\title{
ERJ
}

open research

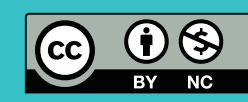

\section{Primary care and the COVID-19 pandemic: Schrödinger's cat}

\author{
To the Editor:
}

We read with interest the article by Tomos et al. [1] reporting on the low death rates during the coronavirus disease 2019 (COVID-19) pandemic crisis in Greece. The authors underlined the importance of primary care in the management of suspected and mild cases and the reduction of inappropriate hospital referrals [1]. With good intentions, the authors offered their own view by stating that the key issue for the reported "Greek success story" is that the primary healthcare system is based on specialist doctors and not general practitioners [1]. They also suggested an algorithm that will help physicians to stratify the risk of infection, highlighting the importance of telemedicine [1]. We also support the usefulness of this conceptual framework. For large population groups that mainly benefit from rural primary care, limited access to private services can be an additional benefit.

Greece has experienced a prolonged period of serious financial and social crisis [2]. Emerging priorities in health policy have been scheduled within austerity measures drafted as memorandum amendments. Greek policy was to focus on reform efforts with the aim of improving national health services and strengthening primary care services [2], in conjunction with health expenditure control and social support.

Currently our country is being affected by the crisis brought about by the COVID-19 pandemic before it has recovered from the previous economic crisis. Interestingly, complex "epi-epidemic" factors throughout all countries' sectors have been globally reported to strongly influence the epidemic burden in communities [3]. In Greece, such a discussion may result in extra support for the primary healthcare services, which in cooperation with the public health services can contribute to the epidemiological surveillance of the pandemic, as well as to the management of asymptomatic or oligo-symptomatic patients [4] and their families.

This new lived experience can be used to also feed the design and implementation of programmes to buffer individual and social frailty. At the same time, it is expected to lead to the interconnection of primary healthcare services with those of social care, by launching evidence-based initiatives for the education and preparation of future primary care provider generations [4].

General practitioners, through continuity of care, can provide more primary care services and can achieve greater management efficiency for their patients [5]. In a recent editorial from the UK, experts in primary care reported that if continuity continues to be weakened, patient safety will be "thinned", organisational inefficiency will grow and collusion of anonymity (when no one takes personal full responsibility for a patient) will become prevalent [5]. Psychosocial factors such as fear and anxiety that individuals and families have experienced during the pandemic have also been addressed [6]. In this respect, primary care patients can benefit from principles such as empathy [5] and the development of effective patient-physician communication. Remarkably, empathy in general practice has been related to a reduced overall mortality in patients with highly demanding conditions such as diabetes $[5,7]$.

Many specialists in Greece working at their private practices offer first contact and longitudinal contact care services. Perhaps this happens because access to specialisations and flows are not systematically regulated based on evidence-reported health system needs. According to Tomos et al. [1], medical visits by

@ERSpublications

The \#COVID19 pandemic crisis requires the collaboration of all healthcare providers. Every contribution is welcome to gain time during the phase of "system state" superposition. https://bit.ly/3m4SBxY

Cite this article as: Symvoulakis EK, Anyfantakis D. Primary care and the COVID-19 pandemic: Schrödinger's cat. ERJ Open Res 2021; 7: 00730-2020 [https://doi.org/10.1183/23120541.00730-2020]. 
experienced chest specialists rather than general practitioners was the main reason for the low hospital referral rates for COVID-19. Taking into account this approach, we would like to build on a dilemma by metaphorically appraising the experiment reported by the Austrian physicist Erwin Schrödinger in 1935 [8]. A cat, a flask of poison and a radioactive source are placed in a sealed box. If the radioactive source remains intact and the flask of poison is not shattered, the cat is still alive. During the experiment, the cat is either alive or dead [8]. Theoretically, an observer could open the flask and examine the cat, but such an action might alter the final outcome $[8,9]$.

We can compare the health system to the sealed box, COVID-19 to the flask of poison and primary care to the position of the "poor" cat. Radioactivity could represent metaphorically the disaccording views on health service utility from all perspectives. Someone may state that the cat is likely to be only black or only white. However, for most, the cat is more likely to be of mixed colour. Considering the uncertainty about the state of the cat, it could be reasoned that the colour does not matter in terms of procedure. However, perceptions between different observers can lead to opening of the "box", which means weakening of the collective capacity to handle the ongoing crisis. In Italy, ARMOcIDA et al. [10] recognising health system fragmentation as an important determinant during the pandemic, stated that "in response to emergencies, solid partnerships between the private and public sector should be institutionalised", and that "recruitment of human resources must be planned and financed with a long-term vision".

Our view is that the COVID-19 pandemic requires gentle management. Reversing the weakening in the continuity of care represents the hallmark for patients' safety [5]. Employment of direct phone lines and electronic messaging by general practitioners for their patients together with extending the time of consultation could be useful future adaptations [5]. In Greece, before the COVID-19 pandemic struck, about 100 local health units (TOMY) became operational in urban environments nationwide, but the lack of sufficient numbers of family physicians, financial incentives to ensure adequate recruitment and sustainability of the project over time proved challenging [2]. At a rural level, a more mature general practice driven system has existed for decades [2]. However, promoting a patient-centred curriculum that will empower future general practitioners with compassionate communication skills has emerged as a priority need [6]. The COVID-19 pandemic is not free of "stigmatisation" in terms of personal and family uncertainty, as it is not yet able to predict the long-term effects of social isolation.

This difficult situation can be handled with the collaboration of all healthcare providers. Every contribution is welcome in order to gain time during the phase of "system state" superposition. It is clear that many specialists offer primary care services instead of secondary or tertiary care services in Greece. It goes without saying that their contribution to community well-being should be respected. However, academic dialogue on issues such as human resource planning to address skill mix and core competencies between specialists and general practitioners, inter-professional training, e-health info operational connection throughout the entire health system and systemically driven care coordination can enhance cooperative and harmoniously actioned initiatives [2] without misunderstandings. For doctors, colour is not a reason to open the box.

\section{Emmanouil K Symvoulakis $^{1}$ and Dimitrios Anyfantakis $\odot^{2}$}

${ }^{1}$ Clinic of Social and Family Medicine, University of Crete Faculty of Medicine, Heraklion, Greece.

${ }^{2}$ Primary Care, Primary Health Care Centre of Kissamos, Chania, Greece.

Correspondence: Emmanouil K. Symvoulakis, Clinic of Social and Family Medicine, School of Medicine, University of Crete, Greece, 71003, Heraklion, Crete, Greece. E-mail: symvouman@yahoo.com

Received: 6 Oct 2020 | Accepted: 14 Oct 2020

Conflict of interest: E.K. Symvoulakis has nothing to disclose. D. Anyfantakis has nothing to disclose.

\section{References}

1 Tomos I, Kostikas K, Hillas G, et al. Primary care and COVID-19: cutting the Gordian knot - the Greek experience and algorithm. ERJ Open Res 2020; 6: 00468-2020.

2 Lionis C, Symvoulakis EK, Markaki A, et al. Integrated people-centred primary health care in Greece: unravelling Ariadne's thread. Prim Health Care Res Dev 2019; 20: el13.

3 Symvoulakis EK, Sourvinos G, Spandidos DA, et al. COVID19 pandemic: monitoring spacetime data and learning from global experience. Exp Ther Med 2020; 20: 73.

4 Lionis Christos. 30/09/2020. Let's learn from what we have missed. https://virus.com.gr/quot-as-mathoyme-apoayta-poy-den-eginan-quot/ Date last accessed: October 3, 2020.

5 Gray DP, Freeman G, Johns C, et al. Covid 19: a fork in the road for general practice. BMJ 2020; 370: m3709.

6 Lionis C, Petelos E. Challenges, priorities and tasks for the generalists at the time of COVID-19 pandemic. Eur J Gen Pract 2020; 26: 104-105. 
7 Dambha-Miller H, Feldman AL, Kinmonth AL, et al. Association between primary care practitioner empathy and risk of cardiovascular events and all-cause mortality among patients with type 2 diabetes: a population-based prospective cohort study. Ann Fam Med 2019; 17: 311-318.

8 Trimmer JD. The present situation in quantum mechanics: a translation of Schrödinger's "Cat Paradox" paper. Proc Am Philos Soc 1980; 124: 323-338.

9 Packer M. The parable of Schrödinger's Cat and the illusion of statistical significance in clinical trials. Circulation 2019; 140: 799-800.

10 Armocida B, Formenti B, Ussai S, et al. The Italian health system and the COVID-19 challenge. Lancet Public Health 2020; 5: e253. 